International Journal of Pure and Applied Mathematics

Volume 110 No. $3 \quad$ 2016, 397-406

ISSN: 1311-8080 (printed version); ISSN: 1314-3395 (on-line version)

url: http://www.ijpam.eu

doi: 10.12732 /ijpam.v110i3.1

\title{
ON THE EXISTENCE OF A NON-RELATIVISTIC HERMITIAN TIME OPERATOR
}

\author{
Carringtone Kinyanjui ${ }^{1}$, Dismas S. Wamalwa ${ }^{2} \S$ \\ ${ }^{1,2}$ Department of Physics \\ University of Nairobi \\ Chiromo, Nairobi, 30197-00100, KENYA
}

\begin{abstract}
The existence of a Hermitian time operator is proposed in the framework of non-relativistic quantum mechanics. The Heisenberg equation of motion is shown to yield constant rate of flow of time consistent with classical expectations when Bohr's correspondence principle is invoked. Further, it is shown that a massless free particle with non-zero momentum experiences timelessness in its own frame of reference whereas a negative massive particle perceives forwardness of time. Application to quantum harmonic oscillators show instantaneous transition of electrons between eigenstates.
\end{abstract}

AMS Subject Classification: 81Q12, 81Q60

Key Words: time operator, non-relativistic quantum mechanics, Bohr, Heisenberg equation

\section{Introduction}

In classical mechanics, dynamical relations usually connect time and space coordinates. However, when we move from classical Mechanics to quantum mechanics, the space coordinates are elevated to operators while the time coordinate enters as a parametric quantity. This discrepancy causes the need to search for a time operator in quantum mechanics. Nevertheless, the need for construction

Received: $\quad$ May 25, 2016

Revised: July 22, 2016

Published: November 5, 2016

${ }^{\S}$ Correspondence author (c) 2016 Academic Publications, Ltd. url: www.acadpubl.eu 
of a time operator in quantum mechanics was first rubbished by Pauli [1]. In standard quantum mechanics, measurable properties of a system are usually assigned Hermitian operators [2]. Time is normally taken to be an internal property of quantum systems because no such operator, Hermitian in nature, has been found to be viable in the treatment of quantum systems [1]. However, a year later after Pauli's dismissal of a time operator, Von Neumann [3] considered the viability of such an operator and we quote him:

".....we must admit that this objection points at an essential weakness which is, in fact, the chief weakness of quantum mechanics: its non-relativistic character, which distinguishes the time $t$ from the three space coordinates $x, y, z$ and presupposes an objective simultaneity concept. In fact, while all other quantities (especially those $x, y, z$ closely connected with $t$ by the Lorentz transformation) are represented by operators, there corresponds to the time an ordinary number-parameter $t$, just as in classical mechanics."

Since the time of Neumann, a number of researchers have attempted to construct the time operator in quantum mechanics with various degrees of success [4], [5] and [6].

Pauli's objection for the existence of a viable time operator is based on the non-existence of a self-adjoint time operator conjugate to discrete or semibounded hamiltonian. For a long time, quantum observables have been known to be self-adjoint operators. The lack of self-adjointedness of a time operator was, therefore, taken to mean that time is not an observable. Nevertheless, time assumes dynamical significance during occurence of events and in time-energy uncertainty relation. Furthermore, observables that are not self-adjoint but with positive-operator-valued measures (POVM) have resurrected the quantum time operator problem [7].

The construction of time operators in quantum mechanics has been done using Mandelstam-Tamm and quantization procedures. In this paper, we seek a hybrid approach in developing such an operator without altering the basic mathematical structure and physical postulates of quantum theory by lifting classical mechanics to quantum mechanics in analogy to Einstein's equivalence principle. We hold that time can be, and infact is, a property of systems in quantum mechanics.

\section{Derivation}

Using the correspondence principle [8] that operators have relations analogous to the classical properties they represent, we take account of the kinetic energy 
operator:

$$
\hat{T}=-\frac{\hbar^{2}}{2 m} \frac{\partial^{2}}{\partial x^{2}}
$$

We can equate this to the classical expression of kinetic energy to obtain a velocity operator:

$$
\hat{v}= \pm \frac{i \hbar}{m} \frac{\partial^{2}}{\partial x^{2}} .
$$

From classical Mechanics, we know that velocity and displacement are considered to be vectors while time a scalar. Since we expect the operator to reproduce the results of classical Mechanics in large quantum numbers, and the time operator to be Hermitian in nature, we adopt in our working the negative component of the velocity operator.

\subsection{The Displacement Operator}

We postulate an operator $\hat{D}$ of the form:

$$
\hat{D}=-\frac{\hbar^{2}}{p^{2}} \frac{\partial^{2}}{\partial x^{2}}
$$

The justification we give, for this, is that it yields the classical result for distances when we consider many body systems. Therefore, its expectation value in a state $\psi$ is given by:

$$
<\hat{D}>=\int \psi^{*} \hat{D} \psi d x
$$

If we consider a free particle system such that

$$
\psi=e^{\frac{i(p \mathrm{x}-E t)}{\hbar}}
$$

and set the upper and lower bounds at $b$ and $a$ respectively, we obtain:

$$
<\hat{D}>=\int_{a}^{b} e^{\frac{i(E t-p \mathrm{x})}{\hbar}}\left(-\frac{\hbar^{2}}{p^{2}} \frac{\partial^{2}}{\partial x^{2}}\right) e^{\frac{i(p \times-E t)}{\hbar}} d x=b-a .
$$

Since $x$ is in units of distance, $b-a$ is in units of distance consistent with the classical description of the same Bohr's correspondence principle. Now for a particle of mass $m$ and momentum eigenvalues $p$, we can use equations (2) and (3) to write down the one dimensional Hermitian time operator as:

$$
\hat{t}=\frac{\hbar m}{i p^{2}} \frac{\partial}{\partial x} \text {. }
$$




\section{Consistency Tests}

We now seek to test the operator in equation (6) for consistency with standard quantum theory. We check it for hermicity, consistency with Bohr's correspondence principle and Heisenberg's equation of motion.

\subsection{Proof of Hermicity}

If $t$ is Hermitian, then

$$
<\hat{t} f|g>=<f| \hat{t} g>
$$

or

$$
<\hat{t} f \mid g>=\int_{a}^{b}\left(\frac{i \hbar m}{p^{2}} \frac{\partial f}{\partial x}\right)^{*} g d x .
$$

Assuming $\mathrm{f}$ and $\mathrm{g}$ vanish at $\mathrm{a}$ and $\mathrm{b}$ respectively;

$$
<\hat{t} f \mid g>=\int_{a}^{b} \frac{\partial f^{*}}{\partial x}\left(\frac{-i \hbar m}{p^{2}} g\right) d x .
$$

Integrating this equation by parts and assuming $f, g$ are analytic functions of $x$ so that $f$ and $g$ vanishes at minus and plus infinity respectively, we have:

$$
<\hat{t} f \mid g>=\int_{a}^{b} f\left(\frac{i \hbar m}{p^{2}} \frac{\partial g}{\partial x}\right)^{*} d x .
$$

this implies that

$$
<\hat{t} f|g>=<f| \hat{t} g>
$$

hence, $\hat{t}$ is Hermitian.

\subsection{Test of Consistency with Bohr's Correspondence Principle}

In large quantum numbers, we expect quantum theory to reproduce results of classical mechanics according to Bohr's correspondence principle [8]. The expectation value for time for a free particle is given as:

$$
\begin{aligned}
& <\hat{t}>=\int_{a}^{b} \psi^{*} \frac{-i \hbar m}{p^{2}} \frac{\partial}{\partial x} \psi d x \\
& <\hat{t}>=\frac{i \hbar m}{p^{2}} \int_{a}^{b} \psi^{*} \frac{\partial}{\partial x} \psi d x
\end{aligned}
$$




$$
\begin{aligned}
<\hat{t}> & =\frac{m}{p} \int_{a}^{b} e^{\frac{i(E t-p \mathrm{x})}{\hbar}} e^{\frac{i(p \mathrm{x}-E t)}{\hbar}} d x \\
<\hat{t}> & =\frac{m(b-a)}{p} \\
<\hat{t}> & =\frac{b-a}{v}=t .
\end{aligned}
$$

In large quantum numbers, the value of $p$ tends towards $m v$.

\subsection{Application of Heisenberg's Equation of Motion to the Hermitian Time Operator}

Heisenberg's [9] equation of motion is:

$$
\frac{d<\hat{A}>}{d t}=i \hbar[\hat{A}, \hat{H}]+\frac{\partial \hat{A}}{\partial t}
$$

where $\hat{A}$ is a linear operator and $\hat{H}=p^{2} / 2 m+V(x, t)$. We seek to use equation (9) to help us understand the flow of time in non-relativistic quantum systems. By inspection, $d<\hat{t}>/ d t=0$ i.e., the expected value for time in a physical process does not change. Equation (9) can be written in terms of the time operator as:

$$
\frac{d}{d t}<\hat{t}>=i \hbar[\hat{t}, \hat{H}]+\frac{\partial \hat{t}}{\partial t} .
$$

If we consider a one dimensional linear harmonic oscillator at zero potential whose state is time-independent, it is easy to show from equations (6) and (10) that:

$$
\frac{d<\hat{t}>}{d t}=0
$$

The rate of flow of time is constant in quantum mechanics for the non-relativistic case. This is the quantum mechanical way of stating that time is absolute in the non-relativistic case [10].

\section{Application to Idealised Physical Systems}

\subsection{The Free Particle System}

According to Newton's law of motion [11], a particle under no external forces will take an infinite amount of time to stop or change direction. We seek to 
explore the same situation quantum-mechanically by use of the time operator and standard Sturm-Liouville methods. In Sturm-Liouville theory [12], the expectation value of an observable $\hat{E}$ is represented by:

$$
<\hat{E}>=\int_{-\infty}^{+\infty} \psi^{*} \hat{E} \psi d v=\int_{-\infty}^{+\infty} \psi^{*} \hat{E} \psi d v .
$$

Applying this to the time operator treatment of the free particle in the one dimensional case, using equations (4) and (6) respectively, leads to:

$$
<\hat{t}>=\int_{-\infty}^{+\infty} \psi^{*} \hat{t} \psi d v=\int_{-\infty}^{+\infty} e^{\frac{i(E t-p \times)}{\hbar}}\left(\frac{\hbar m}{i p^{2}} \frac{\partial}{\partial x}\right) e^{\frac{i(p x-E t)}{\hbar}} d x=\infty .
$$

Therefore, the expectation value for time for a free particle is infinite consistent with classical mechanics.

\subsection{A Massless Free Particle}

Consider a massless beam of particles created at $a$ and absorbed at $b$. We can calculate the expectation value for time taken by the particle between emission and absorption using equations (4) and (6) as:

$$
\begin{aligned}
<\hat{t}> & =\int_{a}^{b} \psi^{*} \hat{t} \psi d x \\
& =\frac{m}{p}[x]_{a}^{b}=\frac{m(b-a)}{p} .
\end{aligned}
$$

For $m=0$ and $p \neq 0$;

$$
<\hat{t}>=0
$$

Though we sought to consider the problem non-relativistically, we can see that this is consistent with Einstein's special theory of relativity [11]: $\Delta t^{\prime}=\gamma \Delta t$ where $\gamma$ is the Lorentz factor given by $1 / \sqrt{1-\frac{v^{2}}{c^{2}}}$ so that $\triangle t \rightarrow 0$ when $v \rightarrow c$ as applies to the photon.

\subsection{Consideration of a Free Particle with Negative Rest Mass}

If we now consider a particle with negative rest mass, we may anticipate the operator to yield backward flow of time. However, considering the problem as 
before for a free particle it is easy to show using equations (4) and (6) that:

$$
<\hat{t}>=\int_{-\infty}^{+\infty} \psi^{*} \hat{t} \psi d x=\infty
$$

since $\mathrm{m}$ is negative by definition. The value is still positive. This points to the fact that time can only flow forwards for systems locally. Backward flow of time seems to be a local impossibility.

\section{Application of the Operator to a Real Physical System}

\subsection{The Quantum Harmonic Oscillator}

In calculating the expectation values for quantum harmonic oscillators, we exploit the use of ladder operators [2] to simplify the situation under consideration.

We make use of the relationship between the time operator and the momentum operator to construct a Hermitian time operator in terms of ladder operators. From [12]:

$$
\begin{gathered}
\hat{p} \rightarrow-i \hbar \frac{\partial \psi}{\partial x} \\
\hat{t} \rightarrow \frac{\hbar m}{i p^{2}} \frac{\partial \psi}{\partial x}
\end{gathered}
$$

the momentum operator for a linear harmonic oscillator is written in terms of ladder operators as:

$$
\hat{p}=i \sqrt{\frac{m \hbar \omega}{2}}\left(\hat{a}-\hat{a}^{\dagger}\right)
$$

where $\hat{a}$ is a lowering operator and $\hat{a}^{\dagger}$ is a raising operator. We exploit the relationship between the time operator and the momentum operator in equation (15) to find an expression for time operator in terms of ladder operators. If $\hat{p} \psi=\frac{p^{2}}{m} \hat{t} \psi$, then:

$$
\begin{aligned}
\hat{t} \psi & =\frac{m}{p^{2}} \hat{p} \psi \\
\Rightarrow \hat{t} \psi & =\frac{i m}{p^{2}} \sqrt{\frac{m \hbar \omega}{2}}\left(\hat{a}-\hat{a}^{\dagger}\right) \psi
\end{aligned}
$$

Let us now use this operator to find the expectation value for time in a quantum harmonic oscillator. 


\subsection{Finding the Expectation Value for Time in a Quantum Harmonic Oscillator when Acted on by Ladder Operators}

The expectation value for time in a quantum harmonic oscillator is:

$$
\begin{aligned}
& <\hat{t}>=<n|\hat{t}| n> \\
& <\hat{t}>=<n\left|\frac{i m}{p^{2}} \sqrt{\frac{m \hbar \omega}{2}}\left(\hat{a}-\hat{a}^{+}\right)\right| n> \\
& <\hat{t}>=\frac{i m}{p^{2}} \sqrt{\frac{m \hbar \omega}{2}}\left(<n\left|\hat{a}^{+}\right| n>-<n|\hat{a}| n>\right)
\end{aligned}
$$

Let $\frac{i m}{p^{2}} \sqrt{\frac{m \hbar \omega}{2}}=\alpha$, then:

$$
\begin{aligned}
& <\hat{t}>=\alpha(<n|\sqrt{n+1}| n+1>-<n|\sqrt{n}| n-1>) \\
& <\hat{t}>=\alpha(<n|n+1>\sqrt{n+1}-\sqrt{n}<n| n-1>)
\end{aligned}
$$

Since state $\mathrm{n}$ is orthonormal to state $n+1$ and state $n-1$,

$$
\begin{aligned}
<n \mid n+1> & =\langle n \mid n-1\rangle=0 \text { so that } \\
<t> & =0 .
\end{aligned}
$$

We propose that an interpretation of equation (17) can be found in the description of quantum jumps. This result lends mathematical support to the assumption by Bohr that transitions between states are instantaneous [13]. We use this fact to make a principally testable hypothesis as follows:

Since the operator predicts instantaneous electron transition between states, no time of transition between energy states is expected. Any such times of transitions must be attributed to perturbations in the systems, chief of which is Heisenberg's inequality for energy [12]:

$$
\Delta E \cdot \Delta t \geq \frac{\hbar}{2}
$$

where $\Delta E$ is the energy of the particle after action by a ladder operator. We can use equation (18) to construct a further inequality:

$$
\tau_{J} \leq \Delta t
$$

The time, $\tau_{J}$, as defined by Schulman must be less than or equal to time, $\Delta t$, as a result of Heisenberg's inequality. If inequality in equation (19) is violated, then the time operator does not hold true. 


\section{Conclusion}

In this paper, we have derived the Hermitian time operator for non-relativistic quantum mechanics and checked it for consistency with quantum mechanical formalism. Application to the free particle system has shown that the particle persists in motion for eternity. Backward flow of time is impossible at least locally. This means that quantum mechanics does not require backward flow of time in the explanation of delayed choice experiments. We have furthermore, made a prediction that electron transition between states is instantaneous assuming a time Hermitian operator for the non-relativistic case.

\section{Acknowledgements}

We thank University of Nairobi for financial support of this research work. We also acknowledge Lexy Andati for typing services of this paper.

\section{References}

[1] J.G. Muga, R.S. Mayato, I.L. Egusquiza, Time in quantum mechanics, 1, Lect. Notes Phys., 734 (2008), 66-84, doi: 10-1007/978-3-540-73473-4_3.

[2] O.L. Lange, R.E. Raab, Operator Methods in Quantum Mechanics, Oxford University Press, England (1991), 20-35.

[3] J. von Neumann, Mathematical Foundations of Quantum Mechanics, Princeton University Press, Princeton (1955), 354.

[4] Bogdan Mielnik, and G. Torres-Vega, Time operator: The challenge persists, arXiv:1112.4198.

[5] Jan Hilgevoord, Time in quantum mechanics, American Journal of Physics, 70, No. 3 (2002), 301-306.

[6] Mariano Bauer, A dynamical time operator in Dirac's relativistic quantum mechanics, International Journal of Modern Physics, A 29, No. 06 (2014), 1450036.

[7] Arai, Asao, Mathematical theory of time operators in quantum physics, No. 1609 (2008), 24-35, http://www.kurims.kyoto-u.ac.jp/ kyodo/kokyuroku/contents/pdf/1609-03.pdf

[8] Falkenburg, Brigitte, Compendium of Quantum Physics, Springer, Berlin Heidelberg, (2009), 125-131. doi: 10.1007/978-3-540-70626-7_39.

[9] R. Fitzpatrick, Heisenberg Equation of Motion, http://sci.uokufa.edu.iq/ar/teaching/najimaa/files/lectures/Quantum/containt.pdf, accessed 10th Dec., 2016

[10] R. Rynasiewicz, Newton's Views on Space, Time and Motion, http://plato.stanford.edu/entries/newton-stm/, accessed 10th Dec., 2016

[11] J.W. Jewett, R.A. Serway, Physics for Scientists and Engineers, 7th Edn., Thomson, Belfron California (2008), 103-174, 1121-1223. 
[12] V.K. Jain, Introduction to Quantum Mechanics, Alpha Science International Ltd, Oxford (2010), 26-90, 298-299

[13] L. Chiatti, Is Bohr's challenge still relevant?, arXiv:1412.3447v1. 